\title{
ИССЛЕДОВАНИЕ ВЛИЯНИЯ АНИЗОТРОПИИ ПРОНИЦАЕМОСТИ \\ НА РАЗРАБОТКУ МОДЕЛЬНОГО БЛОКА ТЕРРИГЕННОГО НЕФТЕНАСЫЩЕННОГО КОЛЛЕКТОРА В ХОДЕ ГИДРОДИНАМИЧЕСКИХ РАСЧЁТОВ
}

\author{
Ермеков Роман Игоревич1, \\ trommka@mail.ru
Коровин Михаил Олегович1, korovinmo@hw.tpu.ru

Меркулов Виталий Павлович1, merkulovvp@hw.tpu.ru

\author{
Чернова Оксана Сергеевна', \\ chernovaos@hw.tpu.ru \\ 1 Национальный исследовательский Томский политехнический университет, \\ Россия, 634050, г. Томск, пр. Ленина, 30.
}

\begin{abstract}
Актуальность исследования состоит в существовании проблемы недостаточности информации при сборе и анализе геологических и физико-химических свойств разрабатываемых залежей. В частности, такая проблема касается анизотропии проницаемости, значение которой можно узнать первоначально лишь на основе керновых данных, получаемых в результате бурения с керноотборниками. Зачастую данная операция является затратной для недропользователя. Однако при построении геолого-гидродинамической модели величина анизотропии проницаемости является одной из ключевых характеристик пласта и значительно влияет на его поведение.

В иели данной работы входило провести подбор оптимального значения вертикальной анизотропии на основе доступных данных и предложить варианты по улучшению существующей системы разработки в ходе гидродинамических расчётов анизотропной модели.

Объектом исследования является одно из нефтяных месторождений Томской области, состоящее из терригенных отложений. Особенностью месторождения является его месторасположение в системе сложных локальных поднятий разного порядка. Основной продуктивный коллектор представлен юрскими отложениями, сформировавшимися в регрессивнотрансгрессивную серию осадконакопления и имеющими сложное распределение фильтрационно-емкостных свойств.

Методы: изучение особенностей течения фрюидов в пористой среде, измерение и подбор значения вертикальной анизотропии проницаемости, а также проведение гидродинамических вычислений с учётом предлагаемых улучшений системы разработки.

В результате исследования удалось узнать, что повышение значения вертикальной анизотропии не всегда приводит к увеличению выработки запасов в зоне работы скважины. Так, при значении вертикальной анизотропии 0,5 величина накопленной добычи нефрти составила 1,968 млн м³, в то время как для значения 0,86 эта величина равнялась 1,913. Такой результат связан с геологическими особенностями продуктивного пласта и подчеркивает важность влияния анизотропии проницаемости. Кроме этого, выполнены гидродинамические расчёты на изотропной и анизотропной моделях, позволившие выделить оптимальные варианты оптимизации текущей системы разработки. Коэффициент извлечения нефти для изотропной модели составил 23,4 \%, а для анизотропной модели - 22,8 \% относительно размера извлекаемых геологических запасов. Таким образом, полученная разница в 0,6 \% вновь показывает важность явления анизотропии проницаемости и необходимость как можно бо́льшего количества данных.
\end{abstract}

\section{Ключевые слова:}

Анизотропия проницаемости, масштаб анизотропии, вертикальная анизотропия, гидродинамическое моделирование, система разработки.

\section{Введение}

В классическом представлении под фильтрационной анизотропией проницаемости понимается соотношение горизонтальной $\left(k_{h}\right)$ и вертикальной проницаемостей $\left(k_{v}\right)[1,2]$. Хорошо известно и установлено, что, как правило, горизонтальная составляющая параметра преобладает по отношению к вертикальной [3]. Более того, множество данных отражают, что изменение фильтрационных свойств имеет разный характер проявления также в самой горизонтальной плоскости [4].

Такие результаты привели к необходимости пересмотра основного закона движения жидкостей и газов в пористой среде - уравнения Дарси. Дарси открыл свой закон в 1856 г., проводя серию экспериментов по изучению движения потока жидкости при фильтрации через поровое пространство цилиндрического песчаного образца породы. В качестве силы, действующей на флюид, он использовал гравитацию (в современных лабораториях используются насосы). В результате своего эксперимента Дарси получил экспериментальную зависимость, выражающую связь скорости фильтрации флюида от градиента давления. Его дифференциальная форма для одномерного случая может быть представлена в следующем виде:

$$
V=\left(\frac{Q}{A}\right)=-\frac{k}{\mu} \frac{\Delta P}{L}=-\frac{k}{\mu}\left(\frac{\partial P}{\partial x}\right),
$$


где $V$ - скорость фильтрации (м/с); $k$ - коэффициент (проницаемость), являющийся динамической характеристикой пористой среды и независящий от свойств флюида (мД); $\Delta P$ - перепад давления, созданный на концах образца (Па); $\mu$ - абсолютная вязкость флюида (мПа·с).

Минус в формуле показывает, что направление движения флюида является противоположным положительному направлению градиента давления. Ocновные допущения, принимаемые для вывода данного выражения:

а) ламинарный или «ползущий» режим течения флюида; б) порода на сто процентов насыщена одним флюидом; в) флюид химически не взаимодействует с породой; г) порода является гомогенной и изотропной.

Позже было установлено, что такую же зависимость можно получить путём осреднения уравнения Навье-Стокса, являющегося одним из важнейших уравнений гидродинамики.

Из курса математического анализа известно, что градиент есть вектор, который своим направлением указывает направление наибольшего роста физической величины. Градиент может быть записан как векторная операция в следующем виде:

$$
\bar{\nabla}=\frac{\partial}{\partial x} \bar{i}+\frac{\partial}{\partial y} \bar{j}+\frac{\partial}{\partial z} \bar{k}
$$

где $\bar{i}, \bar{j}, \bar{k}$ - единичные векторы. Данная операция может быть проведена над скалярной величиной, такой как давление, $p$ :

$$
\bar{\nabla} P=\frac{\partial P}{\partial x} \bar{i}+\frac{\partial P}{\partial y} \bar{j}+\frac{\partial P}{\partial z} \bar{k} .
$$

Из формулы можно видеть, что величина $\bar{\nabla} P$ есть в действительности вектор градиентов давления в направлении $x, y, z$ (рис. 1). Тогда её можно переписать в следующей форме:

$$
\bar{\nabla} P=\left(\begin{array}{l}
\frac{\partial P}{\partial x} \bar{i} \\
\frac{\partial P}{\partial y} \bar{j} \\
\frac{\partial P}{\partial z} \bar{k}
\end{array}\right) .
$$

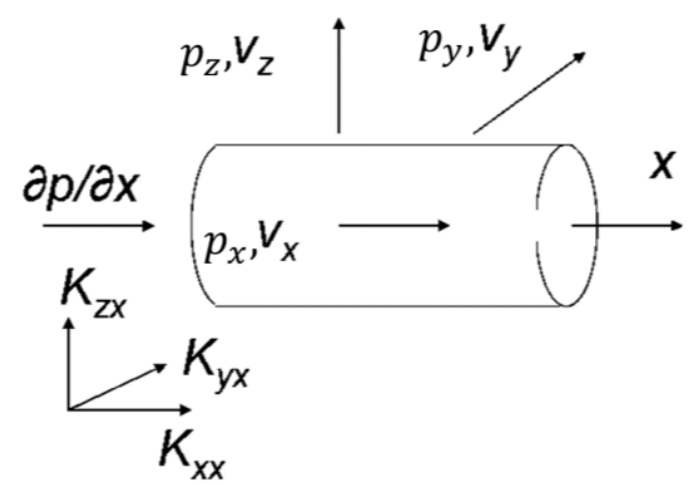

Рис. 1. Направления векторов скоростей фильтрации и градиентов давления [3. С. 7]

Fig. 1. Directions of filtration rates vectors and pressure gradients [3. P. 7]
Из описанного выше следует, что проницаемость есть также величина векторная и её изменение происходит в трёх взаимно ортогональных направлениях. Это даёт возможность описывать данную величину как тензор, имеющий третий порядок, и рассматривать его как прямоугольный параллелепипед:

$$
\bar{k}=\left[\begin{array}{lll}
k_{x x} & k_{x y} & k_{x z} \\
k_{y x} & k_{y y} & k_{y z} \\
k_{z x} & k_{z y} & k_{z z}
\end{array}\right],
$$

где $k_{x x}, k_{y y}, k_{z z}$ - элементы главной диагонали матрицы, являющиеся ортогональными к плоскостям параллелепипеда и называемые нормальными составляющими вектора проницаемости; оставшиеся шесть значений есть касательные компоненты векторов проницаемости и называются симметричными элементами (рис. 2).

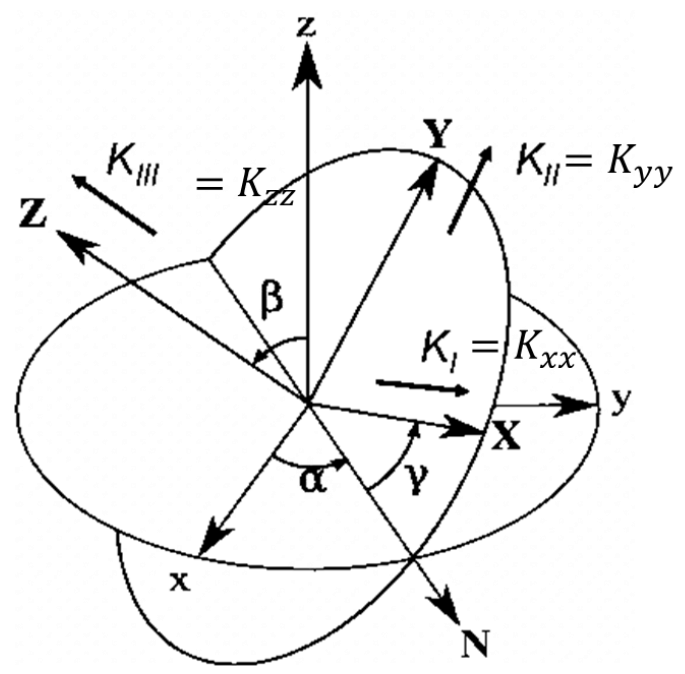

Pис. 2. Направления нормальных и тангенциальных составляющих тензора проницаемости [3. С. 10]

Fig. 2. Directions of normal and tangential components of permeability tensor [3. P. 10]

Таким образом, произведение проницаемости и градиента давления в законе Дарси могут быть записаны следующим образом:

$$
\begin{gathered}
\bar{k} \cdot \bar{\nabla} P=\left[\begin{array}{lll}
k_{x x} & k_{x y} & k_{x z} \\
k_{y x} & k_{y y} & k_{y z} \\
k_{z x} & k_{z y} & k_{z z}
\end{array}\right] \cdot\left(\begin{array}{l}
\frac{\partial P}{\partial x} \bar{i} \\
\frac{\partial P}{\partial y} \bar{j} \\
\frac{\partial P}{\partial z} \bar{k}
\end{array}\right)= \\
=\left[\begin{array}{l}
k_{x x}\left(\frac{\partial P}{\partial x}\right)+k_{x y}\left(\frac{\partial P}{\partial y}\right)+k_{x z}\left(\frac{\partial P}{\partial z}\right) \\
k_{y x}\left(\frac{\partial P}{\partial x}\right)+k_{y y}\left(\frac{\partial P}{\partial y}\right)+k_{y z}\left(\frac{\partial P}{\partial z}\right) \\
k_{z x}\left(\frac{\partial P}{\partial x}\right)+k_{z y}\left(\frac{\partial P}{\partial y}\right)+k_{z z}\left(\frac{\partial P}{\partial z}\right)
\end{array}\right] .
\end{gathered}
$$

Однако описание явления анизотропии с помощью такого математического представления проницаемости пока является крайне затруднительным. Это связано с тем, что явление анизотропии проницаемости 
недостаточно изучено с позиции теории, причин возникновения, механизма проявления связи геологических и петрофизических особенностей и практической значимости для разработки месторождений [5].

\section{Гидродинамические расчёты на анизотропной модели}

Создание анизотропной модели требовало применения данных по величинам анизотропии [6-10]. Первым шагом в этой части стало изучение влияния вертикальной анизотропии $\left(\mathrm{a}_{\mathrm{z}}\right)$ на поведение модели пласта. На основании доступных данных было выбрано три возможных варианта значений вертикальной анизотропии, при этом значения латеральной анизотропии были найдены на основе трёх источников данных и использованы в дальнейшем в расчётах [11-13]. Результаты расчётов вариации вертикальной анизотропии отражены в табл. 1.

Здесь $k$ - исходный куб проницаемости модели (permeability cub of model); $\mathrm{Q}_{\mathrm{o}}$ - накопленная добыча нефти (cumulative oil production); $\mathrm{Q}_{\mathrm{w}}$ - накопленная добыча воды (cumulative water production); $\mathrm{Q}_{\text {inj }}-$ накопленная закачка воды (cumulative water injection); КИН - коэффициент извлечения нефти (oil recovery factor). Интересным фактом здесь является то, что накопленные добыча нефти и закачка воды при $\mathrm{a}_{\mathrm{z}}=0,5 \mathrm{k}$ имеют бо́льшие величины, чем при $\mathrm{a}_{\mathrm{z}}=0,86 \mathrm{k}$. Предполагается, что такой результат связан с особенностями строения резервуара, такими как присутствие высокопроницаемых пропластков в нижней части пласта. Как результат, это привело к тому, что фронт заводнения двигался выше при $\mathrm{a}_{\mathrm{z}}=0,5 \mathrm{k}$, чем при $\mathrm{a}_{\mathrm{z}}=0,86 \mathrm{k}$, и, таким образом, вытеснял большее количество нефти (рис. 3-5). Анализ результатов показывает, что значение $\mathrm{a}_{\mathrm{z}}=0,5 \mathrm{k}$ является более надежной оценкой [14-16]. Величина латеральной анизотропии в двух направлениях была взята равной $0,67 k$ и $1,48 k$ соответственно.

Таблица 1. Результаты вариащии вертикальной анизотропии

Table 1. Results of vertical anisotropy variation

\begin{tabular}{|r|c|c|c|}
\hline \multicolumn{4}{|c|}{ Сценарий, млн м ${ }^{3} /$ Scenario, ММ m } \\
\hline \multirow{2}{*}{$a_{z}$} & $\begin{array}{c}\text { Пессимистичный } \\
\text { Pessimistic }\end{array}$ & $\begin{array}{c}\text { Наиболее вероятный } \\
\text { Most likely }\end{array}$ & $\begin{array}{c}\text { Оптимистичный } \\
\text { Optimistic }\end{array}$ \\
\cline { 2 - 4 } & $a_{z}=0,1 k$ & $a_{z}=0,5 k$ & $a_{z}=0,86 k$ \\
\hline $\mathrm{Q}_{\mathrm{o}}$ & 1,849 & 1,968 & 1,913 \\
\hline $\mathrm{Q}_{\mathrm{w}}$ & 1,350 & 1,281 & 0,893 \\
\hline $\mathrm{Q}_{\mathrm{inj}}$ & 3,082 & 3,142 & 2,655 \\
\hline $\begin{array}{c}\mathrm{K} \text { ИН } \\
\mathrm{ORF}\end{array}$ & 0,139 & 0,148 & 0,144 \\
\hline
\end{tabular}

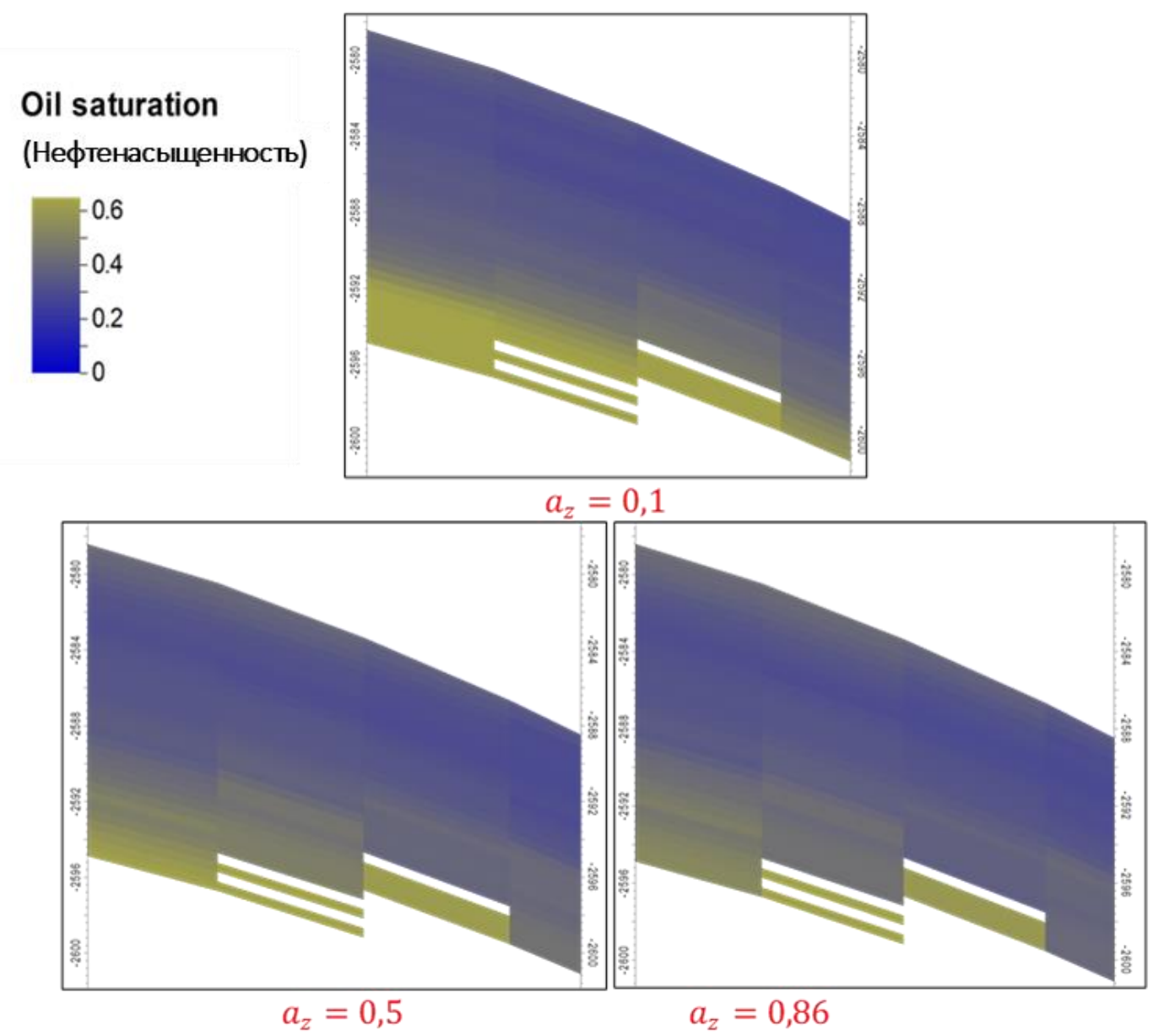

Pис. 3. Сравнение фронтов продвижения воды между тремя вариантами значений вертикальной анизотропии

Fig. 3. Comparison of waterflood fronts between three variants of vertical anisotropy values 


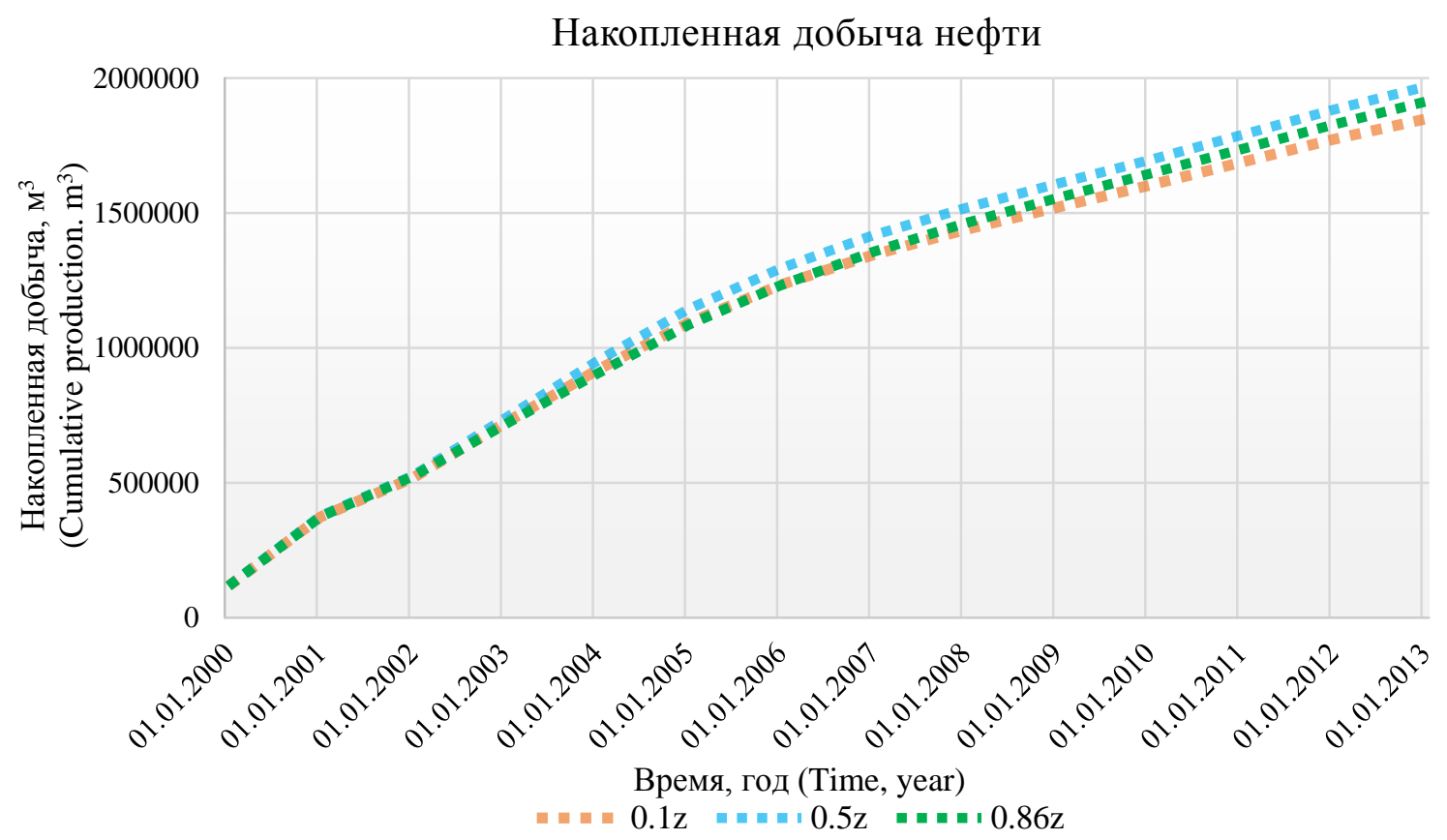

Pис. 4. Сравнение накопленной добычи нефти между тремя вариантами значений вертикальной анизотропии Fig. 4. Comparison of cumulative oil production between three variants of vertical anisotropy values

\section{Наколпенная закачка воды}

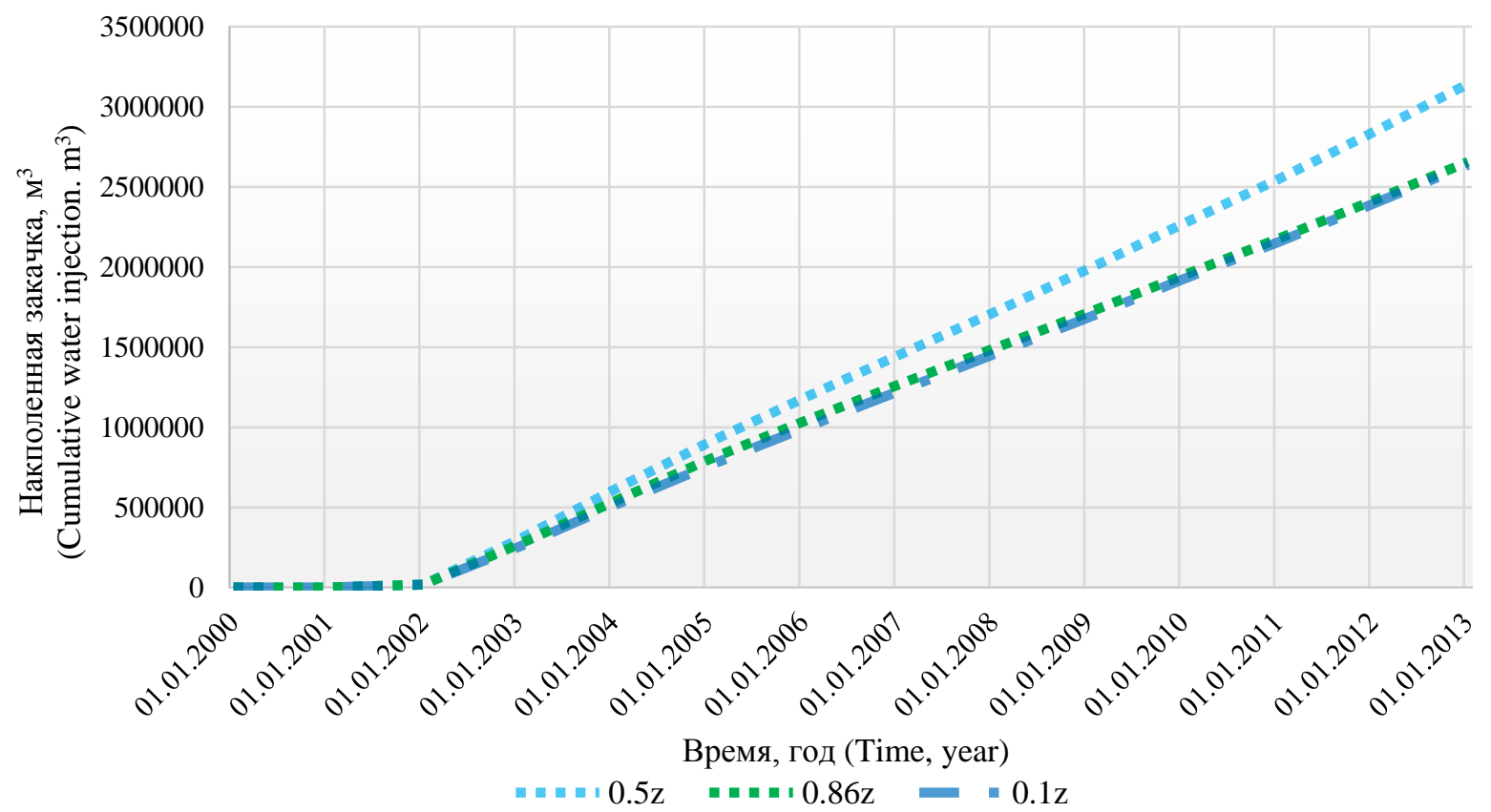

Pис. 5. Сравнение накопленной закачки воды между тремя вариантами значений вертикальной анизотропии

Fig. 5. Comparison of cumulative water injection between three variants of vertical anisotropy values

\section{Оптимизация системы разработки}

Для улучшения системы разработки рассматривалась модернизация существующих скважин. Для проведения этих улучшений было выделено три группы скважин (рис. 6).

Группа A (Group A) состоит из скважин D3, D4, D5, D6, D7, D8, D9, D10, D11, D12, D13. Данная группа скважин соответствует региону проведения трассерных исследований. Из результатов трассерных исследований известно, что эта зона имеет высокопроницаемые фильтрационные каналы северо-восточного простирания, поэтому характеризуется повышенной обводненностью продукции [17]. Однако она также имеет высокие значения величин остаточных запасов. Так 
как бурение дополнительных добывающих скважин в обводненной зоне в данном случае неразумно, наиболее привлекательным вариантом улучшений стало бурение боковых стволов (БС, sidetrack) [18]. Хорошо известно, что бурение боковых или горизонтальных стволов вдоль фильтрационных каналов увеличивает область дренирования скважины без резкого обводнения [19]. В данном случае направление стволов совпадало с направлением улучшенных фильтрационных свойств, т. е. под азимутальным углом $45^{\circ}$. Как показали расчёты, наиболее эффективным и выгодным стало бурение БС в скважинах D5, D8 и D12. Длина каждого ствола составила $300 \mathrm{M}$.

Группа Б (Group B) состоит из скважин D14, D18 и D19. Выделенная территория характеризуется низкими значениями проницаемости. Несмотря на это, она содержит высокие значения остаточных запасов и является наиболее выгодным местом для оптимизации системы разработки с помощью бурения БС. По результатам расчётов, оптимальная длина стволов составила $300 \mathrm{~m}$.

Группа В (group C) состоит из скважин D1 и D15. Здесь бурение БС было эффективно лишь в скважине D1. Скважина D15 имела достаточно высокие значения обводненности в своей области дренирования, что уменьшало эффективность бурения БС.
Кроме описанных мер, были изучены опции по проведению гидроразрыва пласта (ГРП, fracturing). Из проделанной работы удалось выявить, что наиболее эффективным образом операция ГРП была проведена в новых пробуренных добывающих скважинах D23 и D27. Проведение операции в других добывающих скважинах приводило либо к резкой обводненности продукции, либо требовало дальнейшего анализа. Таким образом, все выше предложенные меры были включены в гидродинамические расчёты изотропной и анизотропной моделей на срок продолжительностью 5 лет. Результаты расчётов представлены в табл. 2.

Таблица 2. Сравнение изотропной $и$ анизотропной модели с улучшенной системой разработки

Table 2. Comparison of isotropy and anisotropy models with improved development system

\begin{tabular}{|c|c|c|}
\hline \multirow{2}{*}{$\begin{array}{c}\text { Параметры } \\
\text { разработки } \\
\begin{array}{c}\text { Development } \\
\text { parameters }\end{array}\end{array}$} & \multicolumn{2}{|c|}{$\begin{array}{c}\text { Результаты расчётов в млн м } \\
\text { The calculation results in } \mathrm{MM} \mathrm{m}^{3}\end{array}$} \\
\cline { 2 - 3 } & $\begin{array}{c}\text { Изотропная } \\
\text { модель } \\
\text { Isotropy model }\end{array}$ & $\begin{array}{c}\text { Анизотропная } \\
\text { модель } \\
\text { Anisotropy model }\end{array}$ \\
\hline $\mathrm{Q}_{\mathrm{o}}$ & 3,119 & 3,069 \\
\hline $\mathrm{Q}_{\mathrm{w}}$ & 6,204 & 5,237 \\
\hline $\mathrm{Q}_{\mathrm{inj}}$ & 9,716 & 8,745 \\
\hline КИН & 0,234 & 0,228 \\
\hline
\end{tabular}

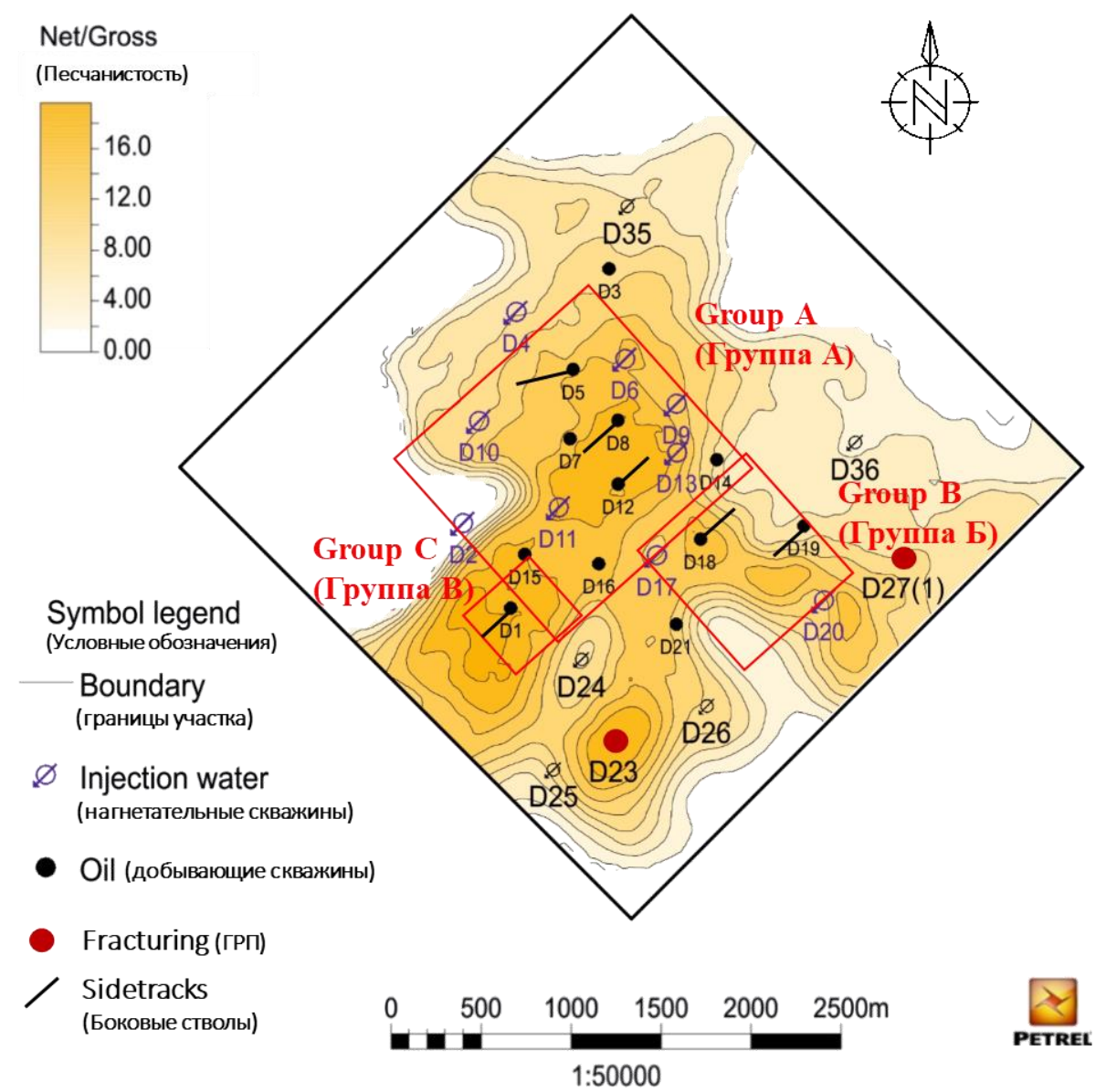

Pис. 6. Карта с указанием выделенных групп скважин - скважин с БС и ГРП

Fig. 6. Map showing selected well groups - sidetracks and wells with fracturing 
Из результатов видно, что параметры изотропной модели оказались выше параметров анизотропной модели. Такой результат указывает на то, что упущение из модельного построения анизотропии может приводить к завышению параметров разработки. Это, в свою очередь, может вызвать превышение ожидаемой добычи и привести к неправильным экономическим расчётам. Данное обстоятельство вновь подтверждает значительное влияние анизотропии проницаемости на разработку.

\section{Заключение}

Как показали результаты вычислений, повышение значения вертикальной анизотропии не всегда приводит к увеличению выработки запасов фронтом завод-

\section{СПИСОК ЛИТЕРАТУРЫ}

1. Permeability anisotropy and its relations with porous medium structure / J.B. Clavaud, A. Maineult, M. Zamora, P. Rasolofosaon, C. Schlitter // Journal of geophysical research. - 2008. - V. 113. P. 1-10.

2. Панков М.В. Анализ разработки Крапивинского нефтяного месторождения // Центр профессиональной переподготовки специалистов нефтегазового дела: отчёт. - Томск: Изд-во НИ ТПУ, 2004. - 425 c.

3. Меркулов В.П., Краснощекова Л.А. Исследование пространственной литолого-петрофизической неоднородности продуктивных коллекторов месторождений нефти и газа // Известия Томского политехнического университета. - 2002. - Т. 305. № 6. - C. 296-303.

4. Tyvand P.A., Storhaug A.R.F. Two-dimensional flow in a porous medium with general anisotropy // Journal of Department of Mathematical Sciences and Technology (Norwegian University of Life Sciences). - 2004. - V. 2. - P. 1-12.

5. Литолого-петрофизическая анизотропия песчано-глинистых коллекторов нефтяных месторождений / В.П. Меркулов, Д.В Александров, Л.А. Краснощекова, Ю.Я. Ненахов // Научнотехнический вестник ЮКОС. - 2004. - № 10. - С. 33-36.

6. Abedi R., Clarke P.L. Modeling of rock inhomogeneity and anisotropy by explicit and implicit representation of microcracks $/ / 52^{\text {nd }}$ U.S. Rock Mechanics/Geomechanics Symposium. - Seattle, Washington, USA, 17-20 June, 2018. - P. 1-11.

7. Zhang Z., Du J. Reservoir characterization using perforation shots: Anisotropy and attenuation // SEG International Exposition and Annual Meeting. - Anaheim, California, USA, 14-19 October, 2018. - P. 2982-2986.

8. Kolbikov S., Kuznetsova Y., Smirnov A. Method of anisotropy modeling and its application to hydrodynamic simulation // SPE Russian Petroleum Technology Conference. - Moscow, Russia, 15-17 October, 2018. - P. 1-9.

9. Makhenko R.Y., Tarokh A. Anisotropy in the undrained pore pressure response of rock // $52^{\text {nd }}$ U.S. Rock Mechan- нения в зоне дренирования добывающих скважин. Поэтому весьма важным являются как источники данных по значению вертикальной анизотропии, так и проведение анализа по подбору её оптимальной величины для гидродинамических расчётов. Анализ вариантов улучшения разработки позволил выделить три группы скважин по критерию наиболее выгодного и рационального геолого-технологического мероприятия (ГТМ): бурение боковых стволов и проведение операции ГРП. Все выбранные ГТМ оказали существенное влияние на продуктивность скважин и внесли большой вклад в конечный профиль чистого дисконтированного денежного потока (NPV - Net Present Value)

ics/Geomechanics Symposium. - Seattle, Washington, USA, 1720 June, 2018. - P. 40-51.

10. Liu S., King M.J. Improved calculation of effective permeability for pore network models using the diffuse source methodology // $81^{\text {st }}$ EAGE Conference and Exhibition. - London, England, UK, 3-6 June, 2019. - P. 1-19.

11. Коровин М.О. Специализированный анализ керна для изучения анизотропии коллекторов нефти и газа // Геофизика. 2014. - T. 324. - № 1. - C. 87-92.

12. Gray D.H., Fatt I., Bergamini G. The effect of stress on permeability of sandstone cores // Society of petroleum engineers' journal. 1963. - V. 3 - P. 95-99.

13. Wannell M.J., Colley N.M., Halford F.R. The use of a new technique to determine permeability anisotropy // SPE journal's. 1993. - V. 9 - P. 489-495.

14. Cosan A. Measuring permeability anisotropy: the latest approach // Oilfield Review, 1994, vol. 6, no. 4, pp. 24-35.

15. Carpenter C. Better permeability estimation from wireline formation testing // Journal of Petroleum Technology. - February, 2018. - V. 70. - P. 66-68.

16. Pan Y., Medhat M.K., Wayne N. Fieldwide determination of directional permeabilities using transient well testing // SPE Reservoir Evaluation \& Engineering. - May, 2019. - V. 22. - P. 1-11.

17. Жабрев И.П., Стуканогов Ю.А. Зависимость нефтеотдачи от направления вытеснения нефти водой из анизотропных по проницаемости пластах // Геология нефти и газа. - 1992. - № 8. - C. 34-36.

18. Al-Hadrami H.K., Teufel L.W. Influence of permeability anisotropy and reservoir heterogeneity on optimization of infill drilling in naturally fractured tight-gas Mesaverde sandstone reservoirs, San Juan Basin // SPE Low Permeability Reservoirs Symposium. Denver, U.S.A., March 12-15, 2000. - P. 1-12.

19. Батлер Р.М. Горизонтальные скважины для добычи нефти, газа и битумов. - М.; Ижевск: Институт компьютерных исследований, НИЦ «Регулярная и хаотическая динамика», 2010. $536 \mathrm{c}$.

Поступила 14.11.2019 2.

\section{Информация об авторах}

Ермеков Р.И., аспирант направления геологии, разведки и разработки полезных ископаемых Инженерной школы природных ресурсов Национального исследовательского Томского политехнического университета.

Коровин М.O., кандидат геолого-минералогических наук, инженер лаборатории геологии месторождений нефти и газа Инженерной школы природных ресурсов Национального исследовательского Томского политехнического университета.

Меркулов В.П., кандидат геолого-минералогических наук, ведущий эксперт Центра подготовки и переподготовки специалистов нефтегазового дела Национального исследовательского Томского политехнического университета.

Чернова O.C., доктор геолого-минералогических наук, ведущий эксперт Центра подготовки и переподготовки специалистов нефтегазового дела Национального исследовательского Томского политехнического университета. 


\title{
UDC 004.942.001.57
}

\section{INVESTIGATION OF PERMEABILITY ANISOTROPY IMPACT ON DEVELOPMENT OF BLOCK OILFIELD OF TERREGINOUS OIL-SATURATED RESERVOIR DURING SIMULATION}

\author{
Roman I. Ermekov1, \\ trommka@mail.ru \\ Mikhail 0. Korovin'1, \\ korovinmo@hw.tpu.ru \\ Vitaliy P. Merkulov 1 , \\ merkulovvp@hw.tpu.ru \\ Oksana S. Chernova1, \\ chernovaos@hw.tpu.ru \\ 1 National Research Tomsk Polytechnic University,
30, Lenin avenue, Tomsk, 634050, Russia.
}

\begin{abstract}
The relevance of the research lies in the problem of insufficient information in collection and analysis of geological and physical-chemical properties of the developed reservoirs. In particular, such problem concerns permeability anisotropy, the value of which can initially be realized only on the basis of cores obtained by core drilling. This is often costly and not beneficial for the subsurface user. However, during construction of geological model, the permeability anisotropy value is one of the key characteristics and significantly effects its behavior.

The aim of this work is to determine the optimal value of vertical anisotropy based on the available data and to propose options to improve the existing development system during simulation of anisotropy model.

The study object is one of oil fields of the Tomsk region, consisting of terrigenous sediments. A feature of the field is its location in the system of complex local lifted blocks of various orders. The main productive reservoir is represented by Jurassic sediments, created in a regressive-transgressive sedimentation series and have a complex distribution of poroperm properties.

Methods: studying the flow characteristics of fluids in the porous medium, measuring and selecting the value of vertical permeability anisotropy, as well as carrying out simulations considering the proposed improvements of the development system.

As a result, it has been learned that increasing the value of vertical anisotropy does not always lead to a growth in production of reserves in the well area. Thus, at a vertical anisotropy value of 0,5, the value of cumulative oil production was $1,968 \mathrm{MM} \mathrm{m}^{3}$, while for 0,86 this value was $1,913 \mathrm{MM} \mathrm{m}^{3}$. This result is related to the geological features of reservoir and emphasizes the importance of the permeability anisotropy effect. Simulations on isotropy and anisotropy models were performed, which allowed highlighting optimal options of optimization of the current development system. The oil recovery factor for the isotropy model was $23,4 \%$ and for the anisotropy model $22,8 \%$ relative to the size of the geological reserves to be extracted. Thus, the resulting $0,6 \%$ difference again shows the importance of the permeability anisotropy phenomenon and the need for as much data as possible.
\end{abstract}

\section{Key words:}

Permeability anisotropy, anisotropy scale, vertical anisotropy, hydrodynamic simulation, development strategy.

\section{REFERENCES}

1. Clavaud J.B., Maineult A., Zamora M. Rasolofosaon P., Schlitter C. Permeability anisotropy and its relations with porous medium structure. Journal of geophysical research, 2008, vol. 113, pp. 1-10.

2. Pankov M.V. Analiz razrabotki Krapivinskogo neftyanogo mestorozhdeniya. Tsentr professionalnoy perepodgotovki spetsialistov neftegazovogo dela: otchet [Analysis of development of the Krapivinsky oilfield. Petroleum Learningl Centre: report]. Tomsk, TPU Publ. house, 2004. 425 p.

3. Merkulov V.P., Krasnoshchekova L.A. Study of spatial lithogenicpetrophysical heterogeneity of productive collectors of oil and gas fields. Bulletin of the Tomsk Polytechnic University, 2002, vol. 305, no. 6, pp. 296-303. In Rus.

4. Tyvand P.A., Storhaug A.R.F. Two-dimensional flow in a porous medium with general anisotropy. Journal of Department of Mathematical Sciences and Technology, 2004, vol. 2, pp. 1-12.

5. Merkulov V.P., Aleksandrov D.V., Krasnoshchekova L.A., Nenakhov Ya.Yu. Lithologic and petrophysical anisotropy of sandshale reservoirs of petroleum fields. Scientific and technical bulletin of YuKOS, 2004, no. 10, pp. 33-36. In Rus.

6. Abedi R., Clarke P.L. Modeling of rock inhomogeneity and anisotropy by explicit and implicit representation of microcracks. $52^{\text {nd }}$ U.S. Rock Mechanics/Geomechanics Symposium. Seattle, Washington, USA, 17-20 June, 2018. pp. 1-11.

7. Zhang Z., Du J. Reservoir characterization using perforation shots: anisotropy and attenuation. SEG International Exposition and An- nual Meeting. Anaheim, California, USA, 14-19 October, 2018. pp. 2982-2986.

8. Kolbikov S., Kuznetsova Y., Smirnov A. Method of Anisotropy Modeling and its Application to Hydrodynamic Simulation. SPE Russian Petroleum Technology Conference. Moscow, Russia, 15-17 October, 2018. pp. 1-9.

9. Makhenko R.Y., Tarokh A. Anisotropy in the undrained pore pressure response of rock. $52^{\text {nd }}$ U.S. Rock Mechanics/Geomechanics Symposium. Seattle, Washington, USA, 17-20 June, 2018. pp. 40-51.

10. Liu S., King M.J. Improved calculation of effective permeability for pore network models using the diffuse source methodology. $81^{\text {st }}$ EAGE Conference and Exhibition. London, England, UK, 3-6 June, 2019. pp. 1-19.

11. Korovin M.O. Specialized analysis of core for study of anisotropy of oil and gas collectors. Bulletin of the Tomsk Polytechnic University. Geophysics, 2014, vol. 324, no. 1, pp. 87-92. In Rus.

12. Gray D.H., Fatt I., Bergamini G. The effect of stress on permeability of sandstone cores. Society of petroleum engineers' journal, 1963, vol. 3, pp. 95-99.

13. Wannell M.J., Colley N.M., Halford F.R. The use of a new technique to determine permeability anisotropy. SPE journal's, 1993, vol. 9, pp. 489-495.

14. Cosan A. Measuring permeability anisotropy: the latest approach. Oilfield Review, 1994, vol. 6, no. 4, pp. 24-35.

15. Carpenter C. Better permeability estimation from wireline formation testing. Journal of Petroleum Technology, February, 2018, vol. 70, pp. 66-68. 
16. Pan Y., Medhat M.K., Wayne N. Fieldwide determination of directional permeabilities using transient well testing. SPE Reser voir Evaluation \& Engineering, May, 2019, vol. 22, pp. 1-11.

17. Zhabrev I.P., Stukanogov Yu.A. Dependence of recovery from direction of oil ousting by warier in beds anisotropic by permeability. Oil and gas Geology, 1992, no. 8, pp. 34-36. In Rus.

18. Al-Hadrami H.K., Teufel L.W. Influence of permeability anisotropy and reservoir heterogeneity on optimization of infill drilling in naturally fractured tight-gas Mesaverde sandstone reservoirs, San Juan Basin. SPE Low Permeability Reservoirs Symposium. Denver, U.S.A., March 12-15, 2000. pp. 1-12.

19. Batler R.M. Gorizontalnye skvazhiny dlya dobychi nefti, gaza $i$ bitumov [Horizontal wells for Oil, gas and bitumen recovery]. Izhevsk, ICI Publ., 2010. 536 p.

Received: 14 November 2019.

\section{Information about the authors}

Roman I. Ermekov, postgraduate, National Research Tomsk Polytechnic University.

Mikhail O. Korovin, Cand. Sc., engineer, National Research Tomsk Polytechnic University.

Vitaliy P. Merkulov, Cand. Sc., expert, National Research Tomsk Polytechnic University.

Oksana S. Chernova, Dr. Sc., expert, National Research Tomsk Polytechnic University. 